Proceedings

\title{
MEMS Enabled Bendable and Stretchable Silicon Circuits ${ }^{\dagger}$
}

\author{
Bram Lips * and Robert Puers \\ KU Leuven, ESAT-MICAS, 3001 Leuven, Belgium; robert.puers@kuleuven.be \\ * Correspondence: bram.lips@esat.kuleuven.be; Tel.: +32-16-327-986 \\ † Presented at the Eurosensors 2018 Conference, Graz, Austria, 9-12 September 2018. \\ Published: 4 December 2018
}

\begin{abstract}
We report the design and implementation of a unique, wafer sized stretchable and bendable monolithic silicon matrix structure. The achieved stretchability allows for simultaneous omnidirectional folding, required to conform the structure to complex curved 3D surfaces. Moreover, it also enables dynamic mechanical deformation of the structure to cope with a moving environment, like e.g., the wall of the heart muscle. Due to the nature of the fabrication process, normal silicon wafer processing can be performed prior to rendering it stretchable. This is first demonstrated by the fabrication of two metal layers on top of silicon, which act as the electrical interconnects of a final flexible and stretchable LED-matrix. Currently, the possibility to postprocess an existing commercial CMOS process is investigated. This would lead to a revolutionary potential of new applications, especially in the medical field, by enabling complex silicon monitoring systems to be linked to organs without hindering them.
\end{abstract}

Keywords: stretchability; dynamic mechanical deformation; silicon post-processing; deep reactive ion etching

\section{Introduction}

At present, the trend toward deformable electronics and their packaging is only starting. With applications in wearable technology, healthcare, mobile devices and the automotive, they will have a profound impact on the current electronic market. But considerable R\&D effort is still required to increase functionality. Most research on deformable electronics at die level is centered on organic materials [1-3]. The main reason being the smaller bending modulus compared to traditional semiconductor materials. However, this advantage comes at the cost of an inherently lower electron mobility and limited thermal stability, leading to less performant electronics. Besides this, these organic materials are often considered as being able to bend the electronics. This is a very narrow definition of deformability. As bending only allows for deformation around one single axis. Bending around several axes simultaneously is a more general definition. This, however, can only be achieved if stretchability is incorporated on top of bendability.

We fabricated a stretchable and bendable monolithic silicon matrix structure. By using silicon as the base semiconductor material, as opposed to organics, low electron mobility and thermal stability are no longer an issue. To render the silicon both stretchable and bendable in all directions, we advanced on a method explained in [4]. In that work, the authors make use of deep reactive ion etching (DRIE) to fabricate a matrix of silicon islands interconnected with thin flexible silicon beams. However, the proposed process requires expensive SOI wafers. We made the process more generally applicable by enabling the use of common $<100>$ wafers. Moreover, the simple silicon beam between the islands, which act as a flexure, is replaced by a silicon serpentine spring. This results in a useful application dependent design tradeoff between stiffness and the silicon area used to fabricate active 
devices. For the first time, electrical interconnects are incorporated on top of the silicon springs by means of standard rigid wafer processing. Enabling the transfer of data and energy from one island to the other. This is demonstrated by the fabrication of a flexible and stretchable LED-matrix.

\section{Materials and Methods}

The proposed stretchable monolithic silicon structure consists of silicon springs interconnecting rigid silicon islands, as shown in Figure 1. Conceptually, these islands can be used to accommodate electronic circuitry and transducers. A two-metal layer process is used to deposit metal tracks on top of the silicon springs, which provide the required electrical interconnects. The main advantage here is that standard rigid silicon wafer processing can be used to fabricate these. No dedicated equipment or processing protocols are needed.

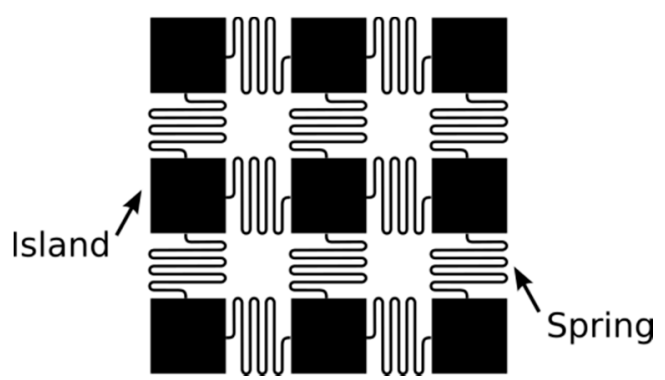

Figure 1. General concept of the stretchable silicon design. Rigid silicon islands are interconnected by silicon serpentine springs.

The complete process flow is depicted in Figure 2, and starts with a standard $<100>$ wafer. It is oxidized and subsequently the first metal layer is deposited through RF sputtering. Patterning is done through lift-off. Next a SiOx inter-layer dielectric is deposited by PVD, this is etched open at specific locations with buffered oxide etch. As such, the first metal layer can be contacted to make bondpads or vias. The second metal layer is deposited and patterned similar to the first one. Now, electrical interconnects are fabricated, and the wafer can be rendered stretchable. Therefore it is first thinned down to a thickness of $100 \mu \mathrm{m}$ by wafer grinding and polishing. The thickness affects the final device rigidity and fracture toughness. A carrier wafer is temporarily bonded on the bottom side of the main wafer. This is done for three main reasons: first it provides some mechanical support during the subsequent DRIE step. Secondly, it protects the DRIE chuck from being damaged by the plasma once the wafer is etched through. And lastly, it keeps all the silicon islands in place after etching. This eases the automatic placement and soldering of discrete LED components. The carrier can be removed again by chemically dissolving the bond layer after soldering. As such the final flexible and stretchable LED-matrix is fabricated.

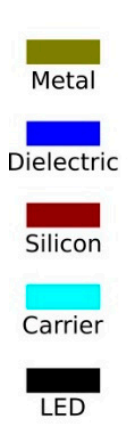

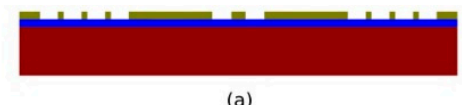

(a)

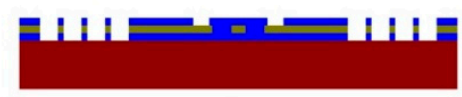

(b)

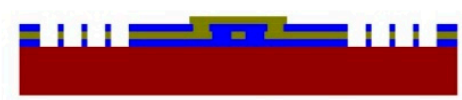

(c)

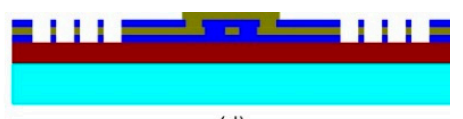

(d)

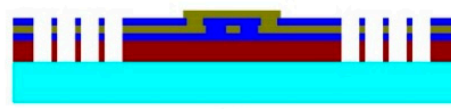

(e)

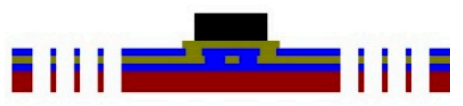

(f)

Figure 2. Overview of the proposed process (a) Si oxidation together with first metal layer deposition and patterning; (b) Inter-layer dielectric deposition and patterning; (c) Second metal layer deposition and patterning; (d) Wafer thinning and carrier mount; (e) DRIE; (f) SMD LED soldering and carrier unmounting. 
Reflow soldering was chosen as the preferred soldering technique and therefore solder paste needed to be applied on appropriate spots. Currently, different techniques have been adopted, with varying results. The main reason for the difficulties being the small footprint of the selected LED $(170 \mu \mathrm{m}$ $\times 250 \mu \mathrm{m}$ contact area with $200 \mu \mathrm{m}$ spacing). Solder paste screen printing was not satisfactory because the mesh size, and therefore the resolution, cannot be chosen independent from the solder paste particle size. Even with the finest solder paste available, too much paste remained stuck to the mesh. Dispensing has been explored as well. However, the finite thickness of the dispense needle in combination with the small distance between the solder dots made it impossible to dispense discrete solder dots. The previously dispensed solder dot is smeared out by the needle shaft upon dispensing of the dot right next to it. The best method explored so far is stencil printing the solder paste with an SU-8 stencil. The stencil is fabricated similar to the one in [5]. This technique works fine as long as the wafer size is limited to 1 or 2 inch. For larger wafers the shrink and warp of the SU-8, caused by internal stresses, makes it impossible to align the stencil accurately. Possibly, this can be resolved by the use of a commercially available metal stencil, which is a method that is being investigated at present.

\section{Results and Discussion}

A close-up image of a fabricated silicon spring, is shown in Figure 3a. The DRIE parameters used are based on [6]. A serpentine design was chosen in order to achieve small spring constants given the limited area between the silicon islands. Dimensions of the beams constituting it, are carefully defined in order to minimize spring constant. These are bound to practical limits imposed by the resistance of the metal tracks running on top of it, and the fracture toughness of the final device. Due to the anisotropic nature of silicon, the orientation of the spring also affects its characteristics. According to calculations and simulations the longitudinal axis of the spring is therefore best placed parallel to the $<100>$-direction. This resulted in a measured spring constant of $24 \mathrm{~N} / \mathrm{m}$ and maximum spring strain values before failure of $100 \%$ to $150 \%$.

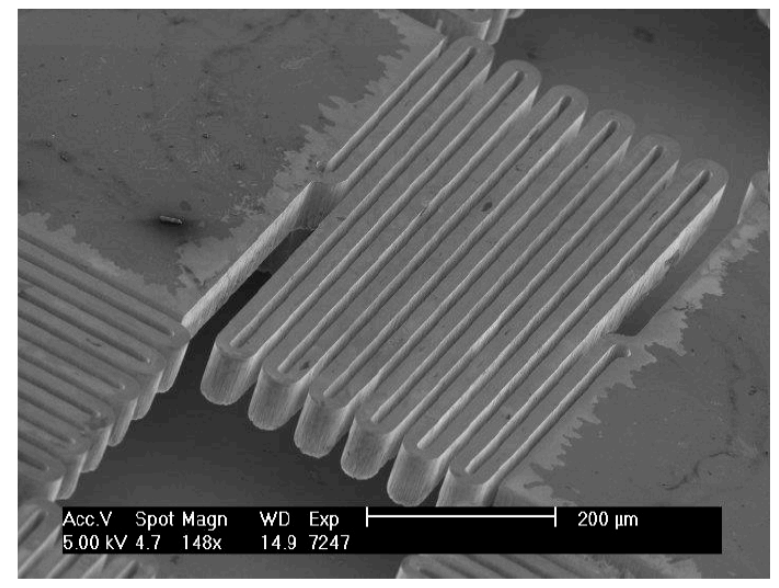

(a)

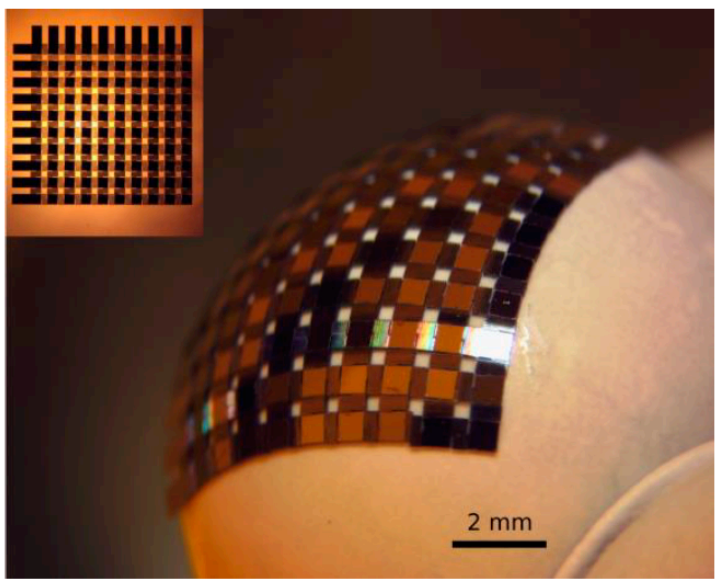

(b)

Figure 3. (a) SEM image of a silicon spring fabricated with the proposed process; (b) The stretchable monolithic device placed on a sphere with a radius of $8 \mathrm{~mm}$, showcasing the possibility to bend it around multiple axes simultaneously. The smaller inset depicts the same silicon before bending.

Figure $3 \mathrm{~b}$ illustrates the capability of the monolithic silicon device to bend around multiple axes simultaneously. The sphere on which the silicon is placed has a radius of $8 \mathrm{~mm}$. Only capillary forces are used to conform the silicon to the underlying shape. Metal tracks incorporated on top of the silicon are used to power the LEDs and are depicted in Figure 4. Measured electrical resistance of a single interconnect is $380 \mathrm{Ohm}$ and remains constant under different spring strain values. 


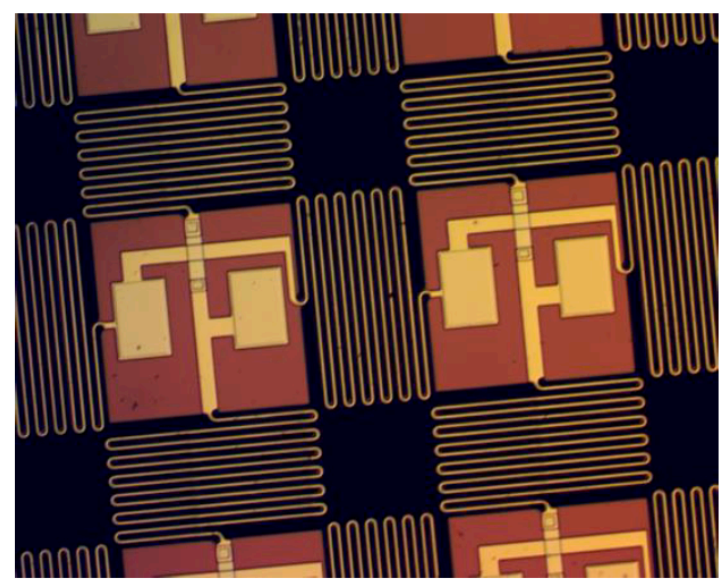

(a)

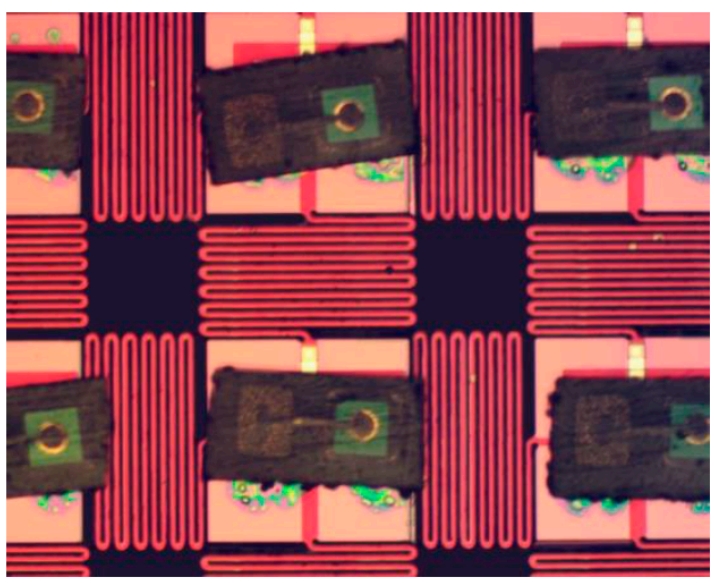

(b)

Figure 4. Microscope image of some silicon islands and springs with metal interconnects running over the springs. Individual islands are $600 \mu \mathrm{m} \times 600 \mu \mathrm{m}$ (a) Before SMD LED's are soldered; (b) After SMD LED's are soldered. The method used to apply the solder, is based on an SU-8 solder stencil.

\section{Conclusions}

A stretchable and bendable silicon circuit was designed and fabricated. This enables simultaneous and repeated bending around multiple axes, necessary to conform the structure to complex curved 3D surfaces. Moreover, a standard rigid $\langle 100\rangle$ wafer processing was used to integrate metal interconnects, prior to rendering it stretchable. These enable data and power transfer between the different islands. Depending on the requirements of the final device, spring dimensions can be optimized. To confirm the wide application range, the first steps towards post-processing an existing commercial CMOS process are planned as a next step.

Funding: The research leading to these results has received funding from the European Research Council under the European Union's Seventh Framework Programme (FP7/2007-2013)/ERC grant agreement No. 340931.

Conflicts of Interest: The authors declare no conflict of interest. The founding sponsors had no role in the design of the study; in the collection, analyses, or interpretation of data; in the writing of the manuscript, and in the decision to publish the results.

\section{References}

1. Park, J.S.; Chae, H.; Chung, H.K.; Lee, S.I. Thin film encapsulation for flexible AM-OLED: A review. Semicond. Sci. Technol. 2011, 26, 034001, doi:10.1088/0268-1242/26/3/034001.

2. Oliveira Hansen, R.M.; Liu, Y.; Madsen, M.; Rubahn, H.G. Flexible organic solar cells including efficiency enhancing grating structures. Nanotechnology 2013, 24, 145301, doi:10.1088/09574484/24/14/145301.

3. Yoon, S.M.; Yang, S.; Byun, C.W.; Jung, S.W.; Ryu, M.K.; Park, S.H.K.; Kim, B.; Oh, H.; Hwang, C.-S.; Yu, B.G. Nonvolatile memory thin-film transistors using an organic ferroelectric gate insulator and an oxide semiconducting channel. Semicond. Sci. Technol. 2011, 26, 034007, doi:10.1088/02681242/26/3/034007.

4. Dinyari, R.; Rim, S.B.; Huang, K.; Catrysse, P.B.; Peumans, P. Curving monolithic silicon for nonplanar focal plane array applications. Appl. Phys. Lett. 2008, 92, 091114, doi:10.1063/1.2883873.

5. Kim, G.; Kim, B.; Brugger, J. All-photoplatic microstencil with self-alignment for multiple layer shadowmask patterning. Sens. Actuat. A-Phys. 2003, 107, 132-136, doi:10.1016/S0924-4247(03)00298-X.

6. Lips, B.; Puers, R. Three step deep reactive ion etch for high density trench etching. J. Phys. Conf. Ser. 2016, 757, 012005, doi:10.1088/1742-6596/757/1/012005.

(C) 2018 by the authors. Licensee MDPI, Basel, Switzerland. This article is an open access article distributed under the terms and conditions of the Creative Commons Attribution (CC BY) license (http://creativecommons.org/licenses/by/4.0/). 